\title{
CHARACTERIZATION OF THE LIMIT LOAD IN THE CASE OF AN UNBOUNDED ELASTIC CONVEX
}

\author{
Adnene Elyacoubi ${ }^{1}$ And Taieb Hadhri ${ }^{1}$
}

\begin{abstract}
In this work we consider a solid body $\Omega \subset \mathbb{R}^{3}$ constituted by a nonhomogeneous elastoplastic material, submitted to a density of body forces $\lambda f$ and a density of forces $\lambda g$ acting on the boundary where the real $\lambda$ is the loading parameter. The problem is to determine, in the case of an unbounded convex of elasticity, the Limit load denoted by $\bar{\lambda}$ beyond which there is a break of the structure. The case of a bounded convex of elasticity is done in [El-Fekih and Hadhri, RAIRO: Modél. Math. Anal. Numér. 29 (1995) 391-419]. Then assuming that the convex of elasticity at the point $\mathrm{x}$ of $\Omega$, denoted by $\mathrm{K}(\mathrm{x})$, is written in the form of $\mathrm{K}^{D}(x)+\mathbb{R I}$, $\mathrm{I}$ is the identity of $\mathbb{R}^{9}$ sym, and the deviatoric component $\mathrm{K}^{D}$ is bounded regardless of $\mathrm{x} \in \Omega$, we show under the condition "Rot $\mathrm{f} \neq 0$ or $\mathrm{g}$ is not colinear to the normal on a part of the boundary of $\Omega$ ", that the Limit Load $\bar{\lambda}$ searched is equal to the inverse of the infimum of the gauge of the Elastic convex translated by stress field equilibrating the unitary load corresponding to $\lambda=1$; moreover we show that this infimum is reached in a suitable function space.
\end{abstract}

Mathematics Subject Classification. 74xx.

Received: February 6, 2004.

\section{The Hencky's Problem for a non-homogeneous elastoplastic STRUCture}

Using the notations and the operators given in [5], the Hencky's problem is given by the following system: find a tensor $\sigma$ and a displacement $u$ such that

$$
\left\{\begin{aligned}
\operatorname{div} \sigma & =\lambda f \quad \text { a.e. in } \quad \Omega \\
\sigma \cdot n & =\lambda g \quad \text { on } \Gamma_{1} \\
u & =u_{0} \text { on } \Gamma_{0} \\
\sigma(x) & =\Pi_{K(x)}\left(A_{(x)}^{-1}(\varepsilon(u)(x))\right)
\end{aligned}\right.
$$

Here:

$$
\varepsilon(u)=\left(\varepsilon_{i j}(u)\right) \quad \text { and } \quad \varepsilon_{i j}(u)=\frac{1}{2}\left(\frac{\partial u_{i}}{\partial x_{j}}+\frac{\partial u_{j}}{\partial x_{i}}\right) \quad \text { for } 1 \leq i, j \leq 3
$$

Keywords and phrases. Elasticity, limit load.

${ }^{1}$ LIM, Polytechnic School of Tunisia. adnene.elyacoubi@ept.rnu.tn; taieb.hadhri@ept.rnu.tn 
$A_{x}^{-1}$ the inverse matrix of $A_{x}, A_{x}$ defined for $\eta=\left(\eta_{i j}\right)_{1 \leq i, j \leq 3} \in \mathbb{R}^{9}$ by:

$$
\left(A_{x}(\eta)\right)_{i j}=\frac{1}{9 K_{0}(x)} \eta_{k k}(x) \delta_{i j}+\frac{1}{2 \mu(x)} \eta_{i j}^{D}
$$

$\mathrm{K}_{0}(x)=\alpha(x)+\frac{2 \mu(x)}{3}$ where $\alpha$ and $\mu$ are the Lamé coefficients.

We suppose that:

$\left(H_{1}\right) \Gamma_{1} \cup \Gamma_{0}=\partial \Omega$ : the boundary of $\Omega$ with $\left(\Gamma_{1}\right) \neq 0$ and the interiors of $\Gamma_{1}$ and $\Gamma_{0}$ satisfy $\Gamma_{1}^{0} \cap \Gamma_{0}^{0}=\emptyset$

$\left(H_{2}\right) K(x)$ is a closed convex part of $\mathbb{R}_{s y m}^{9}$ and $\exists c>0$ such that:

$$
B(0, c) \subset K(x) \text { a.e. in } \Omega \text {. }
$$

Here: $\mathbb{R}_{\text {sym }}^{9}=\left\{X=\left(x_{11}, x_{12}, x_{13}, x_{21}, x_{22}, x_{23}, x_{31}, x_{32}, x_{33}\right), x_{i j} \in \mathbb{R}\right.$ for $1 \leq i \leq 3 ; 1 \leq j \leq 3$ and $\left.x_{i j}=x_{j i}\right\}$ $\left(H_{3}\right) g \in\left(L^{\infty}\left(\Gamma_{1}\right)\right)^{3}, f \in\left(L^{4}(\Omega)\right)^{3}$ such that:

$$
\exists \tilde{g} \in\left(L^{\infty}(\partial \Omega)\right)^{3}, \tilde{g}=g \text { on } \Gamma_{1} \text { and } \int_{\Omega} f \mathrm{~d} x+\int_{\partial \Omega} \tilde{g} \mathrm{~d} \Gamma=0
$$

$\left(H_{4}\right) K(x)=K^{D}(x)+\mathbb{R} I$ and $\exists M>0$ such that:

$$
K^{D}(x) \subset B(o, M) \text { a.e. in } \Omega .
$$

We define the following set $K_{a d}$ :

$$
K_{a d}=\left\{\eta \in\left(L^{2}(\Omega)\right)_{s}^{9} \text { such that } \eta(x) \in K(x) \text { a.e. in } \Omega\right\} .
$$

It is clear that $K_{a d}$ is a closed convex of $\left(L^{2}(\Omega)\right)_{s}^{9}$.

We define now the Quasi-elastic problem:

Find a tensor $\sigma^{e}$ and a displacement $u^{e}$ satisfying:

$$
\left\{\begin{aligned}
\operatorname{div} \sigma^{e} & =f & \text { a.e. in } \Omega \\
\sigma^{e} \cdot n & =\tilde{g} \quad \text { on } \partial \Omega & \\
\sigma^{e}(x) & =\left(A_{(x)}^{-1}\left(\varepsilon\left(u^{e}\right)(x)\right)\right) & \text { a.e. in } \Omega .
\end{aligned}\right.
$$

Referring to [3], the above problem has a solution $\left(\sigma^{e}, u^{e}\right)$, which is unique within a rigid body displacement for $u^{e}$, since $\mathrm{f}$ and g satisfy $\left(H_{3}\right)$; moreover, we have the following proposition.

Proposition 1. We assume that $f$ and $g$ satisfy $\left(H_{3}\right)$, then we have:

$$
\sigma^{e} \in L^{\infty}\left(\Omega, \mathbb{R}_{\text {sym }}^{9}\right)
$$

Proof. According to [3], we have $\sigma^{e} \in\left(W^{1,4}(\Omega)\right)^{9}$ and according to [1] we conclude that: $\sigma^{e} \in L^{\infty}\left(\Omega, \mathbb{R}_{\text {sym }}^{9}\right)$.

\section{Characterization of the limit load $\bar{\lambda}$}

Definition 1. Considering the functional $F$ defined on $V_{1}$ :

$$
\mathrm{V}_{1}=\left\{\eta \in L^{2}\left(\Omega, \mathbb{R}_{\text {sym }}^{9}\right) \text { such that } \operatorname{div} \eta=0 \text { a.e. in } \Omega \text { and } \eta \cdot n=0 \text { on } \Gamma_{1}\right\}
$$

by:

$$
F(\eta)=\mathrm{J}_{K_{a d}}\left(\sigma^{e}-\eta\right)
$$


where, $\mathrm{J}_{K_{a d}}$ is the gauge of $K_{a d}$ defined by:

$$
\mathrm{J}_{K_{a d}}(\alpha)=\inf \{\mathrm{s}>0 \text { such that } \alpha(x) \in \mathrm{s} . \mathrm{K}(\mathrm{x}) \text { a.e. in } \Omega\} .
$$

Then we have:

Proposition 2. The functional $F$ is l.s.c (lower semicontinuous) on $V_{1}$ for the weak topology of $L^{2}\left(\Omega, \mathbb{R}_{\text {sym }}^{9}\right)$ and we have: $F(\eta)=F\left(\eta^{D}\right)$.

Proof. The functional $F$ is l.s.c for the strong topology of $L^{2}\left(\Omega, \mathbb{R}_{\text {sym }}^{9}\right)$ and according to [2] we have $F$ is l.s.c on $V_{1}$ for the weak topology of $L^{2}\left(\Omega, \mathbb{R}_{\text {sym }}^{9}\right)$.

On the other hand $K_{a d}$ is, according to $\left(H_{4}\right)$, unchanged in the direction of the spherical stress, then we have: $F(\eta)=F\left(\eta^{D}\right)$.

Definition 2. The Limit Load $\bar{\lambda}$ is defined in [5] by:

$$
\bar{\lambda}=\sup \left\{\lambda>0 \text { such that } \mathrm{D}_{\lambda} \neq \emptyset\right\}
$$

where

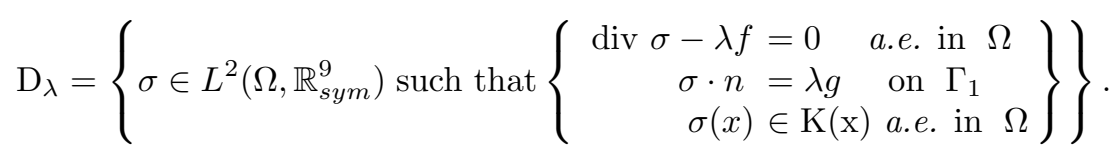

Then we have the following theorems:

Theorem 1. Under the hypotheses $\left(H_{1}\right),\left(H_{2}\right)$ and $\left(H_{3}\right)$ we have:

(i) If $\inf _{\eta \in V_{1}} F(\eta)=0$, then $\bar{\lambda}=+\infty$.

(ii) If $\inf _{\eta \in V_{1}} F(\eta) \neq 0$, then $\bar{\lambda}=\frac{1}{\inf _{\eta \in V_{1}} F(\eta)}$.

Proof.

1. Proof of (i).

We assume that:

$$
\inf _{\eta \in V_{1}} F(\eta)=0
$$

Let $\lambda>0$, and let us show that $\bar{\lambda}>\lambda$.

There exists $\eta \in V_{1}$ such that $F(\eta)<\frac{1}{\lambda}$ and according to (5) we have:

$$
\mathrm{J}_{K_{a d}}\left(\sigma^{e}-\eta\right)<\frac{1}{\lambda} .
$$

Using (6) we obtain:

and then

$$
\left(\sigma^{e}-\eta\right) \in \frac{1}{\lambda} K_{a d}
$$

$$
\lambda\left(\sigma^{e}-\eta\right)(x) \in K(x) \text { a.e. in } \Omega .
$$

On the other hand, we have: $\operatorname{div}\left(\lambda\left(\sigma^{e}-\eta\right)\right)=\lambda\left(\operatorname{div} \sigma^{e}-\operatorname{div} \eta\right)$.

Using (3) and (4) we conclude:

$$
\operatorname{div}\left(\lambda\left(\sigma^{e}-\eta\right)\right)=\lambda f \quad \text { in } \Omega,
$$

and

$$
\lambda\left(\sigma^{e}-\eta\right) \cdot n=\lambda g \text { on } \Gamma_{1} .
$$


From (10)-(12) we obtain: $\lambda\left(\sigma^{e}-\eta\right) \in D_{\lambda}$, and according to (7) it is clear that:

$$
\bar{\lambda}>\lambda .
$$

We finally conclude that:

$$
\bar{\lambda}=+\infty .
$$

2. Using the same idea, we prove (ii).

We now distinguish these two cases:

Theorem 2. Under the hypotheses $\left(H_{1}\right),\left(H_{2}\right),\left(H_{3}\right)$ and $\left(H_{4}\right)$ the following statements (i) and (ii) are equivalent:

(i) $\exists \eta \in V_{1}$ such that $F(\eta)=0$.

(ii) The following problem $\left(P_{2}\right)$ has at least one solution

$$
\left(P_{2}\right)\left\{\begin{array}{l}
\text { Find } \alpha \text { in } W^{1,2}(\Omega) \text { satisfying: } \\
\nabla \alpha=-f \quad \text { in } \Omega \\
\alpha n=-g \quad \text { on } \Gamma_{1}
\end{array}\right.
$$

Proof.

1. Assume there exists $\eta \in V_{1}$ such that $F(\eta)=0$, then according to (5) we have

$$
\mathrm{J}_{K_{a d}}\left(\sigma^{e}-\eta\right)=0,
$$

which implies, using (6):

$$
\inf \left\{s>0 \text { such that }\left(\sigma^{e}-\eta\right)(x) \in s K(x) \text { a.e. in } \Omega\right\}=0 \text {; }
$$

and using $\left(H_{4}\right)$ we have:

$$
\inf \left\{s>0 \text { such that }\left(\sigma^{e}-\eta\right)^{D}(x) \in s K^{D}(x) \text { a.e. in } \Omega\right\}=0,
$$

which implies:

$$
\exists s_{n}>0 ;\left(s_{n}\right)_{n \in \mathbb{N}} \text { independent of } x \text { and such that: }\left\{\begin{array}{l}
s_{n} \underset{n \rightarrow+\infty}{\longrightarrow} 0 \\
\text { and } \\
\left(\sigma^{e}-\eta\right)^{D}(x) \in s_{n} K^{D}(x) \text { a.e. in } \Omega .
\end{array}\right.
$$

Then we can write:

$$
\left(\sigma^{e}\right)^{D}(x)=\eta^{D}(x) \text { a.e. in } \Omega,
$$

and then:

and so:

$$
\eta(x)=\left(\sigma^{e}\right)^{D}(x)+\frac{1}{3} \operatorname{tr} \eta I,
$$

$$
\operatorname{div} \eta=\operatorname{div}\left(\sigma^{e}\right)^{D}+\operatorname{div}\left(\frac{1}{3} \operatorname{tr} \eta I\right) .
$$

But $\eta \in V_{1}$, then $\operatorname{div} \eta=0$, which gives using (3):

$$
\nabla \alpha=-f
$$


where $\alpha=\frac{1}{3}\left(\operatorname{tr} \eta-\operatorname{tr} \sigma^{e}\right)$.

It is clear that $\alpha \in L^{2}(\Omega)$ and $\nabla \alpha \in\left(L^{2}(\Omega)\right)^{3}$ which implies that $\alpha \in W^{1,2}(\Omega)$.

On the other hand, using (4) we obtain:

$$
\eta \cdot n=0 \quad \text { on } \Gamma_{1}
$$

then

$$
\left(\eta^{D}+\frac{1}{3} \operatorname{tr} \eta I\right) \cdot n=0 \quad \text { on } \Gamma_{1}
$$

which can be written using (15) as:

$$
\left(\left(\sigma^{e}\right)^{D}+\frac{1}{3} \operatorname{tr} \eta I\right) \cdot n=0 \quad \text { on } \Gamma_{1}
$$

or

$$
\left(\left(\sigma^{e}\right)+\frac{1}{3}\left(\operatorname{tr} \eta-\operatorname{tr} \sigma^{e}\right) I\right) \cdot n=0 \quad \text { on } \Gamma_{1}
$$

and using (3), we have:

$$
\left(\frac{1}{3}\left(\operatorname{tr} \eta-\operatorname{tr} \sigma^{e}\right) I\right) \cdot n=-g \quad \text { on } \Gamma_{1}
$$

that means:

$$
\alpha \cdot n=-g \quad \text { on } \Gamma_{1}
$$

and using (16) we conclude the first implication.

2. Assume now that $\left(P_{2}\right)$ has at least one solution in $W^{1,2}(\Omega)$, then there exists $\alpha \in W^{1,2}(\Omega)$ such that:

$$
\left\{\begin{array}{l}
\nabla \alpha=-f \quad \text { in } \Omega \\
\text { and } \\
\alpha \cdot n=-g \quad \text { on } \Gamma_{1} .
\end{array}\right.
$$

Then, let

$$
\eta=\sigma^{e}+\alpha I
$$

So, we get:

$$
\eta \in L^{2}\left(\Omega, \mathbb{R}_{\text {sym }}^{9}\right) \text { and } \operatorname{div} \eta=\operatorname{div} \sigma^{e}+\nabla \alpha .
$$

According to (17) and (3) we obtain:

$$
\operatorname{div} \eta=0 \quad \text { in } \Omega .
$$

Let us show now that $\eta \cdot n=0$ on $\Gamma_{1}$.

Let $\phi \in W^{1,2}(\Omega)$ be such that $\phi /\left((\partial \Omega) \backslash \Gamma_{1}\right)=0$, we have for $i \in\{1,2,3\}$

$$
\int_{\Omega} \eta^{i} \nabla \phi \mathrm{d} x=-\int_{\Omega} \operatorname{div} \eta^{i} \phi \mathrm{d} x+\int_{\Gamma_{1}} \eta^{i} \cdot n \cdot \phi \mathrm{d} \Gamma
$$

where $\eta^{i}$ is the vector line of $\eta$.

Using (18) and (20) we obtain:

$$
\int_{\Gamma_{1}} \eta^{i} \cdot n \cdot \phi \mathrm{d} \Gamma=\int_{\Omega}\left(\sigma^{e}\right)^{i} . \nabla \phi \mathrm{d} x+\int_{\Omega}(\alpha . I)^{i} \nabla \phi \mathrm{d} x .
$$


Then since $\phi /\left((\partial \Omega) \backslash \Gamma_{1}\right)=0$,

$$
\int_{\Gamma_{1}} \eta^{i} \cdot n \cdot \phi \mathrm{d} \Gamma=-\int_{\Omega}\left(\operatorname{div} \sigma^{e}\right)^{i} \phi \mathrm{d} x+\int_{\Gamma_{1}}\left(\sigma^{e}\right)^{i} \cdot n \cdot \phi \mathrm{d} \Gamma-\int_{\Omega}(\nabla \alpha)^{i} \phi \mathrm{d} x+\int_{\Gamma_{1}}\left(\alpha \cdot n_{i}\right) \phi \mathrm{d} \Gamma .
$$

And then according to (3) and (17) we have:

$$
\int_{\Gamma_{1}}(\eta \cdot n) \phi \mathrm{d} \Gamma=0
$$

The statements (19)-(21) prove that $\eta \in V_{1}$; moreover, we have:

$$
\left.F(\eta)=F\left(\eta^{D}\right)=F\left(\left(\sigma^{e}\right)^{D}\right)\right)=F\left(\sigma^{e}\right)=0 .
$$

Then

$$
\eta \in V_{1} \text { and } F(\eta)=0
$$

Moreover, we have the following theorem:

Theorem 3. Under the hypotheses of Theorem 2, we have:

$$
\text { If Rotf } \neq 0, \text { then } \inf _{\eta \in V_{1}} F(\eta) \neq 0 .
$$

Proof. Assume that there exists a sequence $\left(\eta_{n}\right)_{n} \in V_{1}$ such that $F\left(\eta_{n}\right) \underset{n \rightarrow+\infty}{\longrightarrow} 0$.

Then, according to (5) we have:

$$
\mathrm{J}_{K_{a d}}\left(\sigma^{e}-\eta_{n}\right) \underset{n \rightarrow+\infty}{\longrightarrow} 0
$$

and then $\exists \alpha_{n}>0\left(\alpha_{n}\right.$ independent of $\left.x\right)$ such that: $\left\{\begin{array}{l}\alpha_{n} \underset{n \rightarrow+\infty}{\longrightarrow} 0 \\ \text { and } \\ \left(\sigma^{e}-\eta_{n}\right)^{D}(x) \in \alpha_{n} K^{D}(x) \text { a.e. in } \Omega .\end{array}\right.$

This gives, according to $\left(H_{4}\right)$ :

$$
\eta_{n}^{D} \underset{n \rightarrow+\infty}{\longrightarrow}\left(\sigma^{e}\right)^{D} \quad \text { a.e. in } \Omega
$$

and

$$
\eta_{n}^{D} \underset{n \rightarrow+\infty}{\longrightarrow}\left(\sigma^{e}\right)^{D} \quad \text { in } \mathrm{D}^{\prime}(\Omega)
$$

Then:

which can be written:

$$
\operatorname{div} \eta_{n}^{D} \underset{n \rightarrow+\infty}{\longrightarrow} \operatorname{div}\left(\sigma^{e}\right)^{D} \quad \text { in } \mathrm{D}^{\prime}(\Omega)
$$

$$
\operatorname{div}\left(\eta_{n}-\frac{1}{3} \operatorname{tr} \eta_{n} . I\right) \longrightarrow[n \rightarrow+\infty] \operatorname{div}\left(\sigma^{e}\right)^{D} \quad \text { in } \mathrm{D}^{\prime}(\Omega) ;
$$

but according to (4) we have $\operatorname{div}\left(\eta_{n}\right)=0$, then:

$$
\operatorname{div}\left(-\frac{1}{3} \operatorname{tr} \eta_{n} . I\right) \underset{n \rightarrow+\infty}{\longrightarrow} \operatorname{div}\left(\sigma^{e}\right)^{D} \quad \text { in } \mathrm{D}^{\prime}(\Omega)
$$

or also

$$
\nabla\left(-\frac{1}{3}\left(\operatorname{tr}\left(\eta_{n}-\operatorname{tr} \sigma^{e}\right)\right) \longrightarrow[n \rightarrow+\infty] \operatorname{div}\left(\sigma^{e}\right) \quad \text { in } \mathrm{D}^{\prime}(\Omega)\right.
$$


which implies:

$$
\operatorname{Rot} \nabla\left(-\frac{1}{3}\left(\operatorname{tr}\left(\eta_{n}-\operatorname{tr} \sigma^{e}\right)\right) \longrightarrow[n \rightarrow+\infty] \operatorname{Rot}\left(\operatorname{div}\left(\sigma^{e}\right)\right) \quad \text { in } \mathrm{D}^{\prime}(\Omega)\right.
$$

Using $\operatorname{Rot} \nabla \delta=0 \quad \forall \delta \in$ in $\mathrm{D}^{\prime}(\Omega)$ we obtain $\operatorname{Rotf}=0$, which concludes the proof.

Corollary 1. Under the hypotheses $\left(H_{1}\right),\left(H_{2}\right),\left(H_{3}\right)$ and $\left(H_{4}\right)$ we have:

$$
\text { If } \operatorname{Rotf} \neq 0, \text { then } \bar{\lambda}=\frac{1}{\inf _{\eta \in V_{1}} F(\eta)} .
$$

Proof. Results from Theorems 1 and 3.

In Theorem 3 we have characterized the Limit load $\bar{\lambda}$; and we have shown that Rotf $\neq 0$ is a sufficient condition to prove that $\bar{\lambda}=\frac{1}{\inf _{\eta \in V_{1}} F(\eta)}$; but this condition is not always satisfied by the volumic force $\mathrm{f}$. In the case where $\operatorname{Rotf}=0$, we introduce in the following a condition on the boundary force $g$ to show the same characterization of the Limit load.

Theorem 4. Under the hypotheses $\left(H_{1}\right),\left(H_{2}\right),\left(H_{3}\right),\left(H_{4}\right)$ and if we assume that Rot $f \neq 0$ or $g$ satisfies:

$$
C_{g}: \exists B \subset \Gamma_{1}, \text { meas }(B) \neq 0 \text { such that } g \wedge n \neq 0 \text { on } B
$$

(which means that $g$ is not colinear to the normal on B).

Then, we have:

$$
F(\eta) \neq 0 \quad \forall \eta \in V_{1}
$$

Proof. The result is deduced from Theorem 2.

In the following part, we will prove, by adding a condition on the open set $\Omega$, that $\bar{\lambda}=\frac{1}{\inf _{\eta \in V_{1}} F(\eta)}$ under hypothesis Rot $\mathrm{f} \neq 0$ or $\mathrm{g}$ satisfying $C_{g}$ given above and that $\inf _{\eta \in V_{1}} F(\eta)$ is reached on $V_{1}$.

\section{An EXISTEnCE RESUlt ObTAined By EXtension of $\Omega$}

\subsection{Problem obtained by extension of $\Omega$}

We assume that $\Omega$ satisfies:

$$
\left\{\begin{array}{l}
\text { There exists an open set } \Omega_{0} \subset \mathbb{R}^{3} \text { such that: } \\
\Omega \subset \Omega_{0} \\
\Omega_{0} \text { is convex } ; \Omega_{0} \backslash \Omega \text { is connex } \\
\partial \Omega \cap\left(\partial\left(\Omega_{0} \backslash \Omega\right)\right)=\Gamma_{1} \\
\text { and } \\
\forall \phi \in \mathrm{W}^{1,2}(\Omega), \exists \phi_{1} \in \mathrm{W}^{1,2}\left(\Omega_{0} \backslash \Omega\right) \text { such that: } \phi=\phi_{1} \text { on } \Gamma_{1} .
\end{array}\right.
$$

Let now $\left(\eta_{n}\right)_{n}$ be a minimizing sequence of $F(\eta)$ on $V_{1}$ and let $\tilde{\eta}_{n}$ be defined by:

$$
\tilde{\eta}_{n}= \begin{cases}\eta_{n} & \text { in } \quad \Omega \\ 0 & \text { in } \quad \Omega_{0} \backslash \Omega\end{cases}
$$


Then we have the following results:

Lemma 1. We assume that (23) is satisfied, then we have:

$$
\operatorname{div} \tilde{\eta}_{n}=0 \quad \text { in } \quad \Omega_{0}
$$

Proof. Let $\phi \in \mathrm{D}\left(\Omega_{0}\right)$, we have:

$$
\int_{\Omega_{0}}\left(\operatorname{div} \tilde{\eta}_{n}^{i}\right) \phi \mathrm{d} x=-\int_{\Omega_{0}} \tilde{\eta}_{n}^{i} \nabla \phi \mathrm{d} x
$$

This means:

$$
\int_{\Omega_{0}}\left(\operatorname{div} \tilde{\eta}_{n}^{i}\right) \phi \mathrm{d} x=-\int_{\Omega} \tilde{\eta}_{n}^{i} \nabla \phi \mathrm{d} x-\int_{\Omega_{0} \backslash \Omega} \tilde{\eta}_{n}^{i} \nabla \phi \mathrm{d} x
$$

and according to (24) we obtain:

$$
\int_{\Omega_{0}}\left(\operatorname{div} \tilde{\eta}_{n}^{i}\right) \phi \mathrm{d} x=\int_{\Omega} \operatorname{div} \eta_{n}{ }^{i} \phi \mathrm{d} x-\int_{\partial \Omega}\left(\eta_{n}{ }^{i} \cdot n\right) \phi \mathrm{d} \Gamma .
$$

But $\phi$ belongs to $\mathrm{D}\left(\Omega_{0}\right)$ then $\phi=0$ on $\partial \Omega \backslash \Gamma_{1}$ and $\left(\eta_{n}\right)_{n} \in V_{1}$, and we get:

$$
\int_{\Omega_{0}} \operatorname{div} \tilde{\eta}_{n}^{i} \phi \mathrm{d} x=0, \text { for all } \phi \in \mathrm{D}\left(\Omega_{0}\right) \text { and for all } 1 \leq i \leq 3,
$$

which allows us to conclude:

$$
\operatorname{div} \tilde{\eta}_{n}=0 \quad \text { in } \quad \Omega_{0} .
$$

Remark 1. Let $\left(\eta_{n}\right)_{n \in \mathbb{N}}$ be a minimizing sequence of $F(\eta)$ on $V_{1}$, then we have:

$$
\mathrm{J}_{K_{a d}}\left(\sigma^{e}-\eta_{n}\right) \leq \text { Const. }
$$

Using Proposition 2 and the property $\left(H_{4}\right)$, we obtain:

$$
\left\|\eta_{n}^{D}\right\|_{L^{\infty}\left(\Omega, \mathbb{R}_{s y m}^{9}\right)} \leq \text { Cte for all } n \in \mathbb{N}
$$

Then, let:

$$
\left(\tilde{\eta}_{n}\right)^{D}= \begin{cases}\eta_{n}^{D} & \text { in } \Omega \\ 0 & \text { in } \Omega_{0} \backslash \Omega\end{cases}
$$

We obtain:

$\left(\tilde{\eta}_{n}^{D}\right)_{n}$ is bounded in $L^{\infty}\left(\Omega_{0}, \mathbb{R}_{s y m}^{9}\right)$, then there exists a subsequence of $\left(\tilde{\eta}_{n}^{D}\right)_{n}$ (denoted also $\left.\left(\tilde{\eta}_{n}^{D}\right)_{n}\right)$ and there exists $\sigma_{0} \in L^{2}\left(\Omega_{0}, \mathbb{R}_{\text {sym }}^{9}\right)$ such that:

$$
\left(\tilde{\eta}_{n}^{D}\right) \longrightarrow \sigma_{0} \quad \text { weakly in } \mathrm{七}^{2}\left(\Omega_{0}, \mathbb{R}_{\text {sym }}^{9}\right) .
$$

Lemma 2. Under the hypotheses $\left(H_{1}\right),\left(H_{2}\right),\left(H_{3}\right),\left(H_{4}\right)$ and assuming that $\Omega$ satisfies (23), we have:

$$
\operatorname{Rot}\left(\operatorname{div} \sigma_{0}\right)=0 \quad \text { in } \Omega_{0}
$$

where $\sigma_{0}$ is the element of $L^{2}\left(\Omega_{0}, \mathbb{R}_{\text {sym }}^{9}\right)$ given by $(27)$. 
Proof. Using (27) we get:

$$
\left(\tilde{\eta}_{n}\right)^{D} \underset{n \rightarrow+\infty}{\stackrel{\sigma}{\longrightarrow}} \quad \text { in } \mathrm{D}^{\prime}\left(\Omega_{0}\right)
$$

which implies:

$$
\operatorname{div}\left(\tilde{\eta}_{n}\right)^{D} \underset{n \rightarrow+\infty}{\longrightarrow} \operatorname{div} \sigma_{0} \quad \text { in } \mathrm{D}^{\prime}\left(\Omega_{0}\right)
$$

and

$$
\operatorname{Rot}\left(\operatorname{div}\left(\tilde{\eta}_{n}\right)^{D}\right) \underset{n \rightarrow+\infty}{\longrightarrow} \operatorname{Rot}\left(\operatorname{div} \sigma_{0}\right) \quad \text { in } \mathrm{D}^{\prime}\left(\Omega_{0}\right)
$$

but we have:

Then according to Lemma 1.1, we obtain:

$$
\operatorname{div}\left(\tilde{\eta}_{n}\right)^{D}=\operatorname{div} \tilde{\eta}_{n}-\frac{1}{3} \nabla\left(\operatorname{tr} \tilde{\eta}_{n}\right)
$$

$$
\operatorname{Rotdiv}\left(\tilde{\eta}_{n}\right)^{D}=-\frac{1}{3} \operatorname{rot} \nabla\left(\operatorname{tr} \tilde{\eta}_{n}\right) \quad \forall n \in \mathbb{N} .
$$

Finally, we conclude from (28) that:

$$
\operatorname{Rot}\left(\operatorname{div} \sigma_{0}\right)=0 \text { in } \mathrm{D}^{\prime}\left(\Omega_{0}\right) .
$$

\subsection{An existence result}

Firstly, we begin by the following result:

Lemma 3. Let $\Omega_{0}$ be an open convex set of $\mathbb{R}^{n}$ and let $v \in\left(H^{-1}\left(\Omega_{0}\right)\right)^{3}$ satisfying:

$$
\text { Rot } v=0 \text { in } \Omega_{0}
$$

Then there exists a unique $q \in L^{2}\left(\Omega_{0}\right) / \mathbb{R}$ such that:

$$
\nabla q=v
$$

Proof. See [5].

Remark 2. We remark that $\operatorname{div} \sigma_{0}$ cannot always be equal to 0 and on the other hand we search a stress $\sigma$ which achieves $\inf _{\eta \in V_{1}} F(\eta)$, so we will change the spherical component to have a stress $\sigma$ satisfying $\operatorname{div} \sigma=0$. That is the purpose of the following paragraph.

Lemma 4. Under the hypotheses of Lemma 2 we have:

$$
\exists \sigma \in L^{2}\left(\Omega_{0}, \mathbb{R}_{\text {sym }}^{9}\right) \text { such that }\left\{\begin{array}{lllll}
\operatorname{div} \sigma & = & 0 & \text { in } & \Omega_{0} \\
\sigma^{D} & = & \sigma_{0}^{D} & \text { in } & \Omega_{0} \\
\sigma & = & 0 & \text { in } & \Omega_{0} \backslash \Omega .
\end{array}\right.
$$

Proof. Using Lemmas 2 and 3 we get: $\exists q \in L^{2}\left(\Omega_{0}\right) / \mathbb{R}$ such that :

$$
\operatorname{div} \sigma_{0}=-\nabla q \quad \text { in } \mathrm{D}^{\prime}\left(\Omega_{0}\right) .
$$

And according to (24) and (27), we deduce:

$$
\sigma_{0}=0 \text { on } \Omega_{0} \backslash \Omega \text {, }
$$

then

$$
\operatorname{div} \sigma_{0}=0 \text { on } \Omega_{0} \backslash \Omega \text {. }
$$


This proves that:

$$
\nabla q=0 \quad \text { on } \quad \Omega_{0} \backslash \Omega .
$$

We have, using (23), $\Omega_{0} \backslash \Omega$ is connex, and then:

$$
q=C_{1} \text { on } \quad \Omega_{0} \backslash \Omega \quad\left(C_{1} \in \mathbb{R}\right),
$$

so we can choose $C_{1}=0$.

Then, let $\sigma \in L^{2}\left(\Omega_{0}, \mathbb{R}_{\text {sym }}^{9}\right)$ be defined by:

$$
\sigma=\sigma_{0}+q I
$$

we have

$$
\sigma=0 \text { on } \quad \Omega_{0} \backslash \Omega .
$$

From (30), (32) and (33) we conclude the result.

We can now prove the main theorem:

Theorem 5. Under the hypotheses $\left(H_{1}\right),\left(H_{2}\right),\left(H_{3}\right),\left(H_{4}\right)$ and assuming that $\Omega$ satisfies (23), there exists $\sigma_{1} \in V_{1}$ such that

$$
F\left(\sigma_{1}\right)=\inf _{\eta \in V_{1}} F(\eta)
$$

so the infimum of $F$ is achieved on $V_{1}$.

Proof. Let $\left(\eta_{n}\right)_{n \in \mathbb{N}}$ be a minimizing sequence of $F$ on $V_{1}, \sigma_{0}$ given in the Remark 1 .

Let $\sigma_{1}=\sigma / \Omega$ where $\sigma$ is given by (29); we have:

$$
\sigma_{1} \in\left(L^{2}(\Omega)\right)_{s}^{9},
$$

and:

$$
\operatorname{div} \sigma_{1}=(\operatorname{div} \sigma) / \Omega=0 \quad \text { in } \Omega .
$$

So,

$$
\operatorname{div} \sigma_{1}=0 \text { in } \Omega .
$$

Let us prove that $\sigma_{1} \cdot n=0$ in $\Gamma_{1}$, it is equivalent to prove:

$$
\int_{\Gamma_{1}}\left(\sigma_{1} . n\right) \phi \mathrm{d} \Gamma=0 \forall \phi \in W^{1,2}(\Omega) \text { be such that } \phi /\left(\partial \Omega \backslash \Gamma_{1}\right)=0 .
$$

Then let $\phi_{1} \in W^{1,2}\left(\Omega_{0} \backslash \Omega\right)$ be such that $\phi_{1} / \Gamma_{1}=\phi / \Gamma_{1}$, and let $\tilde{\phi}$ be defined by:

$$
\tilde{\phi}= \begin{cases}\phi & \text { in } \quad \Omega \\ \phi_{1} & \text { in } \quad \Omega_{0} \backslash \Omega .\end{cases}
$$

We have, due to the trace theorem for $\tilde{\phi}$ on the two sides of $\Gamma_{1}=\partial \Omega \cap\left(\partial\left(\Omega_{0} \backslash \Omega\right)\right)$ :

$$
\tilde{\phi} \in W^{1,2}\left(\Omega_{0}\right) .
$$

On the other hand, we have:

$$
\int_{\Omega_{0}} \sigma \cdot \varepsilon(\tilde{\phi}) \mathrm{d} x=\int_{\Omega} \sigma \cdot \varepsilon(\phi) \mathrm{d} x+\int_{\Omega_{0} \backslash \Omega} \sigma \cdot \varepsilon(\tilde{\phi}) \mathrm{d} x
$$


which implies, according to (33):

$$
\begin{aligned}
\int_{\Omega_{0}} \sigma \cdot \varepsilon(\tilde{\phi}) \mathrm{d} x & =\int_{\Omega} \sigma \cdot \varepsilon(\phi) \mathrm{d} x \\
& =-\int_{\Omega}(\operatorname{div} \sigma) \cdot \phi \mathrm{d} x+\int_{\partial \Omega}\left(\sigma_{1} \cdot n\right) \phi \mathrm{d} \Gamma
\end{aligned}
$$

Using $\operatorname{div} \sigma=0$ in $\Omega_{0}$, we obtain:

$$
\int_{\Omega_{0}} \sigma \cdot \varepsilon(\tilde{\phi}) \mathrm{d} x=\int_{\partial \Omega}\left(\sigma_{1} \cdot n\right) \phi \mathrm{d} \Gamma
$$

This means according to (36) that:

$$
\int_{\Omega_{0}} \sigma \cdot \varepsilon(\tilde{\phi}) \mathrm{d} x=\int_{\Gamma_{1}}\left(\sigma_{1} \cdot n\right) \phi \mathrm{d} \Gamma
$$

We have on the other hand:

$$
\int_{\Omega_{0}} \sigma \cdot \varepsilon(\tilde{\phi}) \mathrm{d} x=\int_{\Omega_{0}}(\operatorname{div} \sigma) \cdot \tilde{\phi} \mathrm{d} x+\int_{\partial \Omega_{0}}(\sigma \cdot n) \tilde{\phi} \mathrm{d} \Gamma .
$$

So, using properties (29) and (36), we deduce that:

$$
\int_{\Omega_{0}} \sigma \cdot \varepsilon(\tilde{\phi}) \mathrm{d} x=0
$$

From (37) and (38), we conclude that:

$$
\int_{\Gamma_{1}}\left(\sigma_{1} . n\right) \phi \mathrm{d} \Gamma=0 \quad \forall \phi \in W^{1,2}(\Omega) \text { such that } \phi /\left(\partial \Omega \backslash \Gamma_{1}\right)=0
$$

This proves that:

$$
\sigma_{1} \cdot n=0 \quad \text { on } \Gamma_{1} .
$$

We conclude from (34), (35) and (39) that:

$$
\sigma_{1} \in V_{1} .
$$

Using Proposition 2 and $\left(\eta_{n}{ }^{D}\right)_{n}$ converge to $\sigma_{0}{ }^{D}$ weakly in $L^{2}\left(\Omega, \mathbb{R}^{9}{ }_{\text {sym }}\right)$, we have:

$$
F\left(\sigma_{1}\right) \leq \underline{\lim } F\left(\eta_{n}^{D}\right)
$$

or also:

$$
F\left(\sigma_{1}\right) \leq \inf _{\eta \in V_{1}} F(\eta)
$$

We finally conclude from (40):

$$
F\left(\sigma_{1}\right)=\inf _{\eta \in V_{1}} F(\eta) .
$$

Corollary 2. Under the hypotheses of Theorem 5 we have:

$$
\text { If Rotf } \neq 0 \text { in } \Omega \text { or }(22) \text { is satisfied by } g
$$


then

$$
\exists \sigma_{1} \in V_{1} \text { such that: } \bar{\lambda}=\frac{1}{\inf _{\eta \in V_{1}} F(\eta)}=\frac{1}{F\left(\sigma_{1}\right)} .
$$

Proof. The result is deduced from Theorems 4 and 5.

\section{REFERENCES}

[1] R. Adams, Sobolev Spaces. Academic Press, New York (1975).

[2] H. Brezis, Analyse Fonctionnelle. Masson, Paris (1983).

[3] P.G. Ciarlet, Lectures on the three-dimensional elasticity. Tata Institute of Fundamental Research, Bombay (1983).

[4] H. El-Fekih and T. Hadhri, Calcul des charges limites d'une structure élastoplastique en contraintes planes. RAIRO: Modél. Math. Anal. Numér. 29 (1995) 391-419.

[5] R. Temam, Mathematical Problems in Plasticity. Bordas, Paris (1985).

$\overline{\text { To access this journal online: }}$ www.edpsciences.org 